\title{
Giffordia fuscata (Zan.) Kuck. nov. comb., eine Ectocarpacee mit heteromorphen, homophasischen Generationen
}

\author{
Von Peter Kornmann \\ Aus der Biologischen Anstalt Helgoland, List auf Sylt \\ in der Bundesanstalt für Fischerei \\ (Mit 5 Abbildungen im Text)
}

\section{A. Einleitung}

In einem Vortrag in Edinburgh berichtete ich (KonNMANN 1953) kurz über einige Ergebnisse von entwicklungsgeschichtlichen Untersuchungen an Giffordia fuscata. Diese Ectocarpacee trägt beiderlei Sporangien: plurilokuläre, deren Schwärmer stets die gleichen fädigen Büschel erzeugen und unilokuläre, aus deren Schwärmern eine Generation von mikroskopisch kleinen Pflänzchen hervorgeht. In Übereinstimmung mit der allgemeinen Auffassung lag es nahe, die Zwerggeneration als Gametophyten anzusehen, zumal in ihr immer wieder büschelige Giffordia-Pflanzen mit pluri- und unilokulären Sporangien auftraten. Diese Vermutung hat sich jedoch nicht bestätigt. Niemals konnten Kopulationen zwischen den Zwergpflanzenschwärmern beobachtet werden, vielmehr traten auch in der Zwerggeneration Pflänzchen mit unilokulären Sporangien auf. Ich möchte daraus schließen, daß die beiden Generationen trotz ihrer morphologischen Verschiedenheit Diplonten sind und keine Reduktionsteilung in den unilokulären Sporangien erfolgt.

\section{Giffordia fuscata (Zan.) Kuck. nov. comb.}

Nach Kuckucks noch unveröffentlichten Aufzeichnungen gehört Giffordia ovata (Kjellm.) Kylin (1947) als Synonym zu Ectocarpus fuscatus Zan. Einzelheiten bleiben einer später erscheinenden Mitteilung in der Reihe der Ectocarpaceen-Studien (KUCKUck $\uparrow 1953$ ) vorbehalten. KuckUck sammelte die Art mehrfach bei Helgoland.

\section{B. Kulturversuche}

Mein Untersuchungsmaterial stammt aus einer Rohkultur von Codium fragile, das im Juni 1951 von Helgoland mitgebracht wurde. Darin entwickelte 
sich die Alge in den Wintermonaten und wächst seitdem in Reinkultur in Erdschreiberlösung.

Die fädigen Büschel werden in meinen Kulturen bis zu $3 \mathrm{~cm}$ lang. Die Fäden sind locker einseitswendig oder gegenständig verzweigt (Abb. 1), sie enden in einer aus verlängerten Zellen bestehenden Spitze. Besonders differenzierte Wachstumszonen sind nicht vorhanden. Die plurilokulären Sporangien sitzen ungestielt oder auf kurzer Stielzelle den Fäden einzeln oder paarig auf, mitunter trägt eine Stielzelle auch zwei Sporangien. Die plurilokulären Sporangien sind in den Kulturen oft recht langgestreckt, sie zeigen aber häufig die für die Art kennzeichnende stärker gewölbte Außenwandung. Unilokuläre Sporángien treten nicht mit Regelmäßigkeit auf, sie sitzen im allgemeinen nur einzeln an den basalen Fadenabschnitten zwischen den plurilokulären Sporangien, können aber mitunter auch längere Abschnitte eines Fadens allein

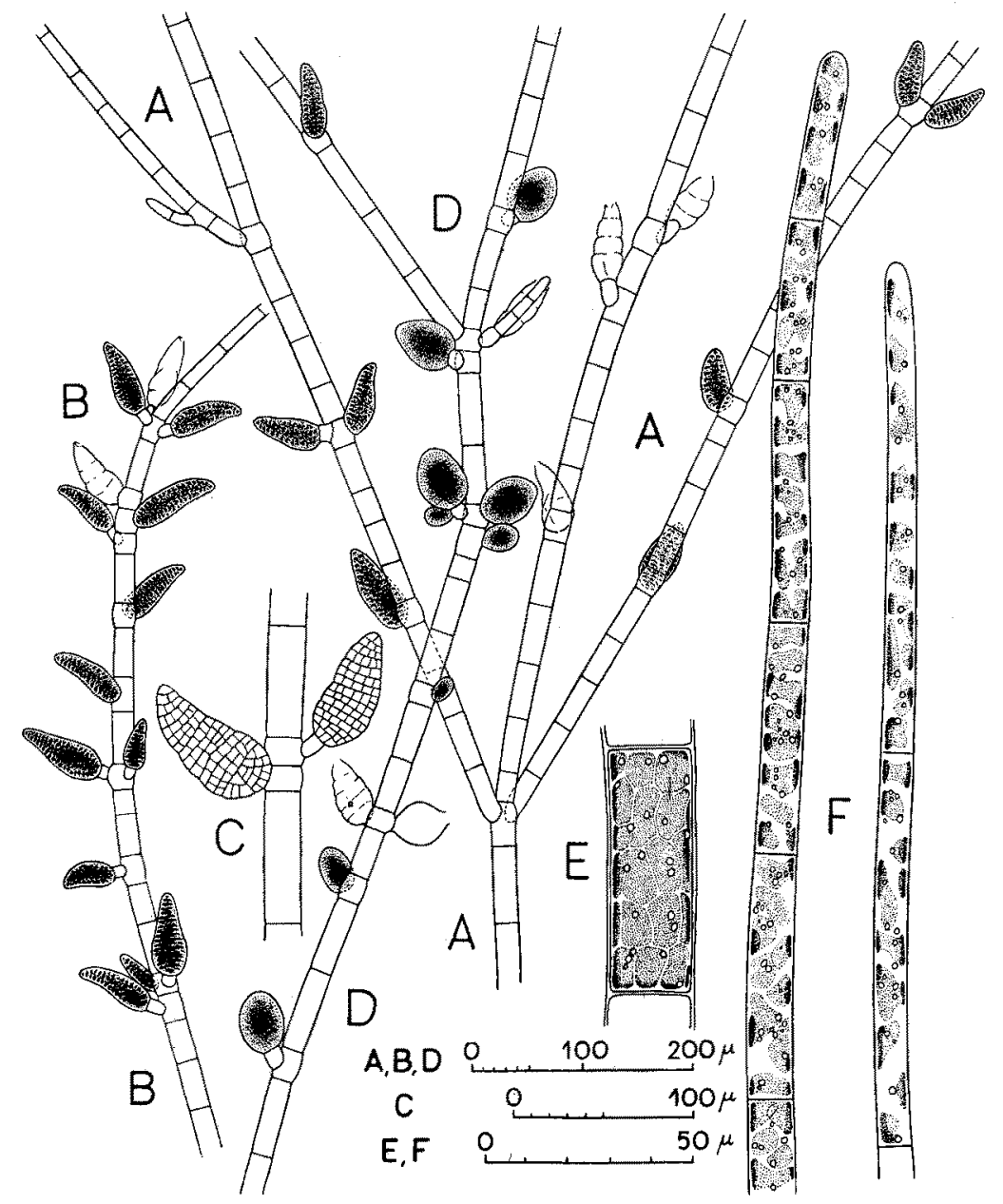

Abb. 1. Giffordia fuscata aus Kulturen.

$A$ Faden mit gegenständiger und einseitswendiger Verzweigung, locker mit plurilokulären Sporangien besetzt. $B$ Fadenstück mit dichtstehenden, ziemlich langgestreckten plurilokulären Sporangien. $C$ Fadenstück mit einem sitzenden und einem einzellig gestielten plurilokulären Sporangium. $D$ Fadenstück überwiegend mit unilokulären Sporangien besetzt. $E$ Fadenzelle mit scheibenförmigen Chromatophoren. $F$ Fäden baarartig in verlängerte Zellen auslaufend. 
einnehmen. Die Art ihrer Anheftung ist die gleiche wie bei den plurilokulären Sporangien.

Fadenstücke mit plurilokulären bzw. unilokulären Sporangien wurden isoliert, um die entsprechenden Ausgangskulturen zu gewinnen. Während das Ausschwärmen der plurilokulären Sporangien keine Schwierigkeiten machte, ging ein großer Teil der unilokulären Sporangien vor der Entleerung zugrunde. Auch entwickelte sich in vielen Fällen nur eine geringe Anzahl ihrer Schwärmer zu fertilen Pflänzchen.

Beiderlei Schwärmer weisen keine morphologischen Besonderheiten auf. Sie sind von gleicher Größe und enthalten einen Chromatophor und einen Augenfleck. Beide Arten von Schwärmern ändern ihr phototaktisches Verhalten in gleicher, eigenartiger Weise: nach dem Verlassen des Sporangiums schwimmen sie zunächst positiv phototaktisch zum Lichtrand, sammeln sich aber nach ganz kurzer Zeit an der dunkelsten Stelle des Gefäßes bzw. eines Tropfens an.

Schon unmittelbar nach dem Festsetzen und während der frühesten Entwicklungsstadien verhalten sich die beiden Schwärmerarten verschieden. Die Schwärmer der plurilokulären Sporangien runden sich nach dem Festsetzen ab und behalten bis zu der bald erfolgenden Keimung ihren rundlichen Umriß bei (Abb. 2, A). Dagegen breiten sich die Schwärmer aus den unilokulären Sporangien unregelmäßig bis sternförmig auf der Unterlage aus (Abb. 3, A). Ebenso verhalten sich auch die Schwärmer der Zwerggeneration, die aus ihnen hervorgeht. Da diese Schwärmer im Gegensatz zu den seltenen Schwärmern aus unilokulären Sporangien stets in reichlicher Menge zur Verfügung standen, wurden sie zu den im nächsten Abschnitt geschilderten Beobachtungen benutzt.

Bringt man Zwergpflanzen-Schwärmer aus einer Kulturschale in einen Tropfen frische Nährlösung, so setzt sich ein großer Teil innerhalb weniger

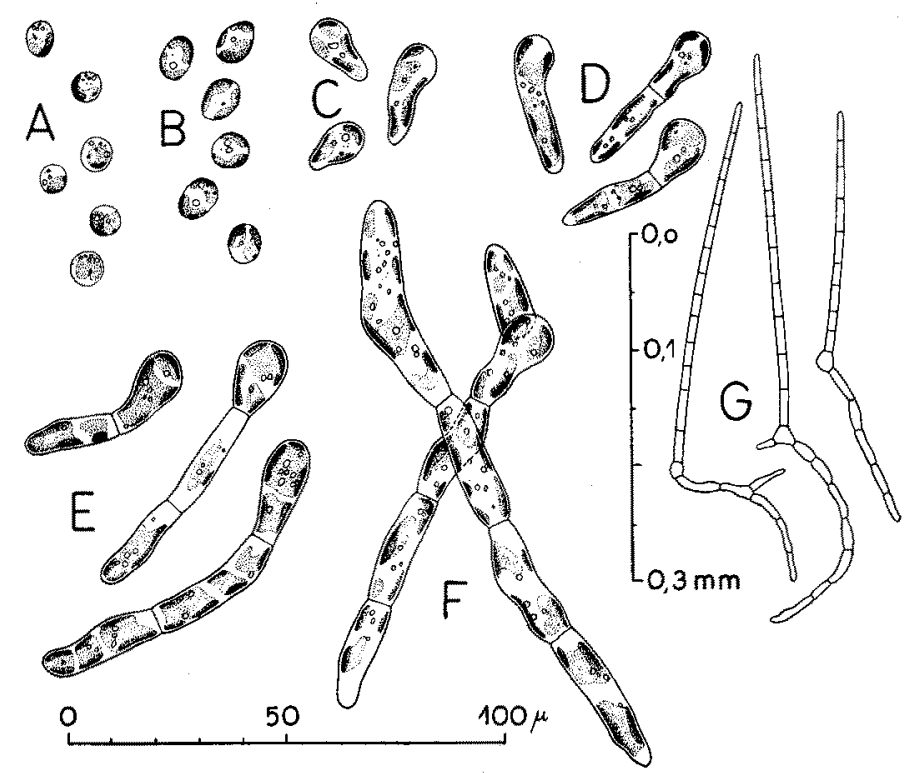

Abb. 2. Giffordia fuscata. Keimung und Entwicklung der Schwärmer aus plurilokulären Sporangien. $A$ Rundliche Embryosporen kurz nach dem Festsetzen der Schwärmer. $B-F$ Keimlinge nach $1,2,3,4$ bzw. 6 Tagen. $G$ Pflänzchen 9 Tage alt. 
Minuten fest. Diese Schwärmcr fallen sofort durch die engen Kreisbahnen auf, die sie beschreiben, während die übrigen Schwärmer sich mehr oder weniger geradlinig bewegen. Die zum Festsetzen bereiten Schwärmer schwimmen auf Kreisbahnen von etwa 40-70 $\mu$ Durchmesser, und zwar stets im umgekehrten Uhrzeigersinne. Diese Bewegung dauert einige Minuten an. Schließlich heftet sich die lange Geißel mit ihrem Ende auf dem Objektträger fest und der so verankerte Schwärmer rotiert noch einige Male in der angegebenen Richtung weiter. Plötzlich wird die lange Geißel verkürzt und damit der Körper des Schwärmers auf den Anheftungspunkt gezogen. Die kurze Geißel ist in diesem Augenblick noch sichtbar, verschwindet aber sogleich. Der Schwärmer setzt sich mit seinem spitzen, farblosen Vorderende auf der Unterlage fest und richtet sich vertikal auf. Schon wenige Minuten später hat er sich zu der unregelmäßig-sternförmigen Embryospore ausgebreitet.

Die Schwärmer aus den plurilokulären Sporangien der büscheligen Giffordia verhalten sich ebenso, breiten sich aber - wie schon erwähnt - nach dem Festsetzen kreisförmig auf der Unterlage aus. Es bleibt zu prüfen, ob vielleicht das oben beschriebene Verhalten der Schwärmer vor ihrem Festheften für die Braunalgen allgemein gültig oder für bestimmte Formenkreise kennzeichnend ist.

Unterschiedliche Embryosporen sind bei manchen anderen Braunalgen bekannt. Sauvageau (1927) brachte die Heteroblastie bei Eudesme zosterae mit einem verschiedenartigen Verhalten der Schwärmer in Zusammenhang. Schwärmer, die sich nach dem Festsetzen innerhalb weniger Minuten zu sternförmigen Embryosporen ausbreiten, ergaben Myrionema-ähnliche Pflänzchen, während sich aus den rundlich ausgebreiteten Embryosporen ectocarpoide Pflänzchen entwickelten. KYLıN (1933), der mit der gleichen Pflanze arbeitete, konnte die Form der Embryospore mit der Dauer des Schwärmens in Beziehung bringen: die rasch sich festsetzenden Schwärmer breiteten sich sternförmig aus, die länger schwärmenden ergaben die rundlichen Embryosporen. In unserem Falle ist die Form der Embryospore bei den beiden Schwärmerarten verschieden, sie ist das früheste Merkmal eines unterschiedlichen Keimungs- und Entwicklungsverlaufes, wie es z. B. auch bei Myrionema strangulans (Sauvageau 1897) der Fall ist.

Die Schwärmer aus den plurilokulären Sporangien der Giffordia-Pflanze entwickeln sich zu einer Generation, die der Ausgangspflanze gleicht. Wie Abb. 2 zeigt, wächst die rundliche Embryospore zu einem Keimschlauch aus, der zu einem Rhizoid wird. Auf diesem erheben sich nach etwa einer Woche aufrechte Fäden, die in die Länge wachsen, sich verzweigen und zuerst immer nur plurilokuläre, später auch unilokuläre Sporangien ausbilden.

Ganz anders verläuft die Entwicklung bei den Schwärmern aus unilokulären Sporangien (Abb. 3). Die sternförmige Embryospore wächst zu einem Fädchen von unregelmäßigen Zellen oder zu einem kleinen Haufen kugelig geballter Zellen heran. Bereits nach einer Woche werden die Pflänzchen fertil und tragen plurilokuläre Sporangien, deren Schwärmer die gleiche Entwicklung wiederholen. Alle 8-10 Tage wird eine neue Zwergpflanzen-Generation fertil.

Die Zwergpflanzen sind untereinander nicht einheitlich, vielmehr lassen sich mehrere Wuchstypen unterscheiden, zwischen denen es Übergangsformen gibt. Die meisten Pflänzchen haben eine fädige Achse, die sich später verzweigt und reichlich plurilokuläre Sporangien trägt (Abb. 4, A). Andere Pflanzen 
bestehen aus kugeligen Zellhaufen, die bei ihrer Reife ringsum von Sporangien eingehüllt werden (Abb. 4, B, C). Schließlich findet man auch Zwergpflanzen, bei denen sich auf dem sporangientragenden Zellfaden oder -knäuel ein kurzer, gerader Sproß erhebt, der aber sein Wachstum bald einstellt (Abb. 4, F, G). Diese geraden Fäden können später auch fertilisiert werden.

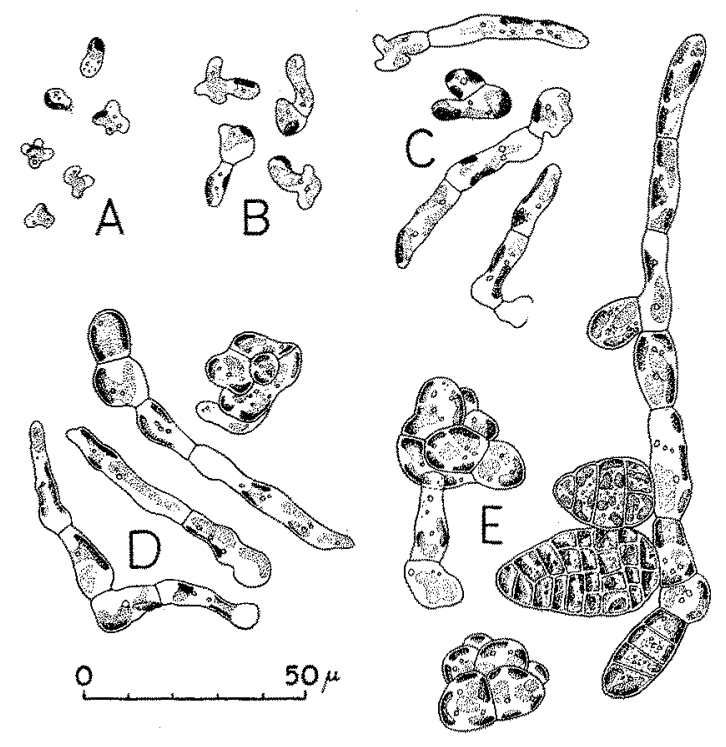

Abb. 3, Giffordia fuscata. Keimung und Entwicklung der Schwärmer aus unilokulären Sporangien. $A$ Unregelmäßige bis sternförmige Embryosporen kurz nach dem Festsetzen der Schwärmer. $B-D$ Keimlinge nach 2, 4 bzw. 6 Tagen. $E$ Zwei knäuelige und ein fädiges Zwergpflänzchen, 9 Tage alt.

Die verschiedenen Typen von Zwergpflanzen treten in der Nachkommenschaft eines einzelnen unilokulären Sporangiums auf. Sie erscheinen auch immer wieder in den Folgegenerationen eines einzelnen $Z$ wergpflänzchens, ganz gleichgültig, von welcher Wuchsform man ausgeht. Durch äußere Einflüsse läßt sich jedoch das Verhältnis der fädigen zu den knäueligen Zwergpflänzchen verschieben: in Nährlösungen mit steigendem Gehalt an Erdabkochung vermindert sich der Anteil der knäueligen Zwergpflanzen.

Wie schon in der Einleitung erwähnt wurde, lag es nahe, in den Zwergpflanzen eine Gametophytengeneration zu vermuten. Damit wäre das vereinzelte Auftreten von fädigen Giffordia-Pflanzen in den $Z_{\text {wergpflanzenkulturen }}$ als Folge einer gelegentlichen Verschmelzung von Gameten zu erklären gewesen. Jedoch blieben alle Versuche erfolglos, in den während zwei Jahren unterhaltenen Kulturen Kopulationen zwischen den Zwergpflanzen-Schwärmern $z u$ beobachten oder morphologische Verschiedenheiten an den plurilokulären Sporangien zu finden. Die Sporangien können zwar eine recht unterschiedliche Größe haben, zeigen aber keine Besonderheiten hinsichtlich ihrer Fächerung.

Die in den Zwergpflanzen-Kulturen auftretenden Giffordia-Pflanzen fallen schon sehr frühzeitig durch ihren geraden aufrechten Faden auf. Sie entwickeln sich zu verzweigten fädigen Pflanzen mit pluri- und unilokulären Sporangien. Wenn sie auch in den einzelnen Kulturen mehr oder weniger 


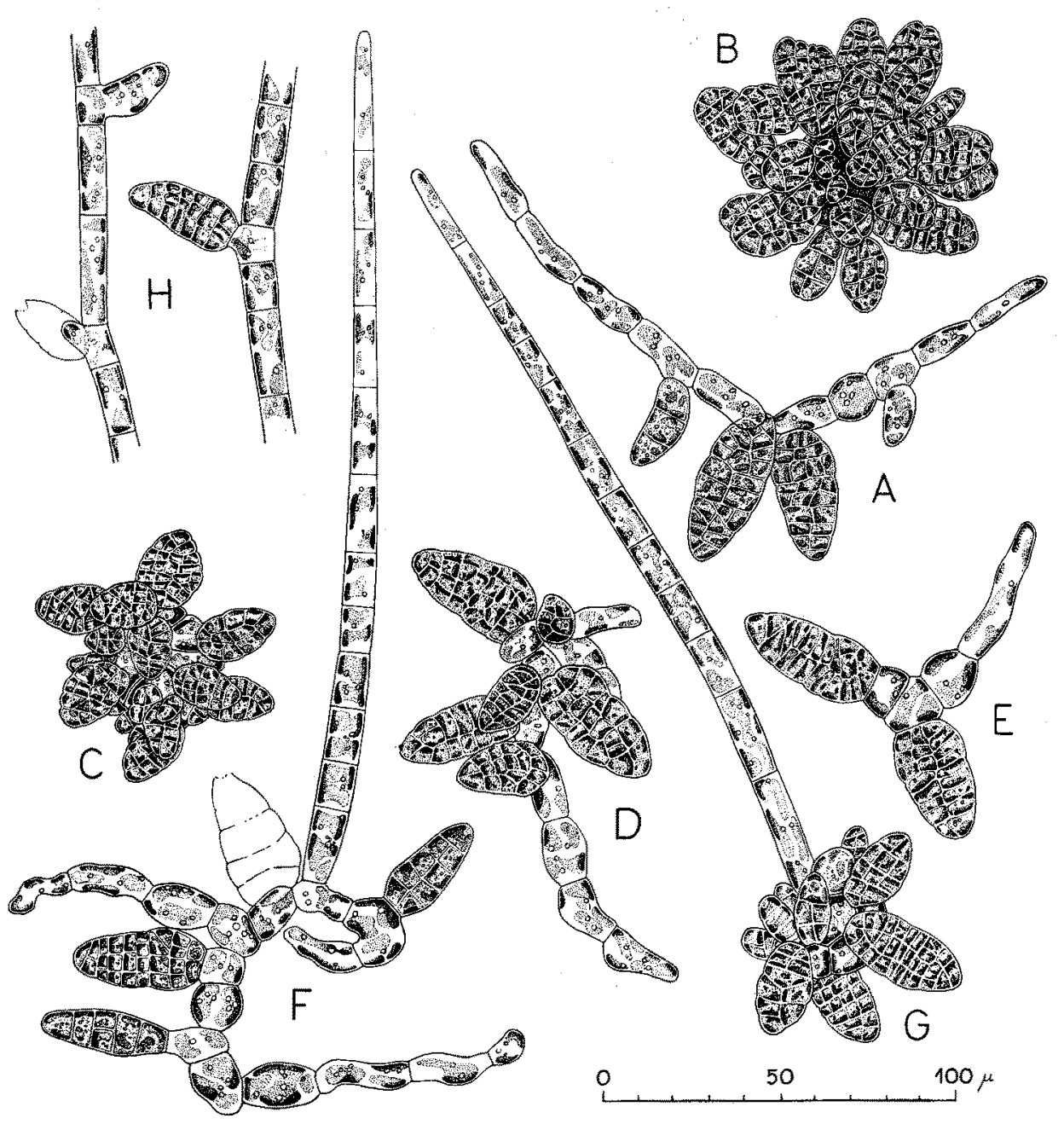

Abb. 4. Giffordia fuscata. Variabilität der Zwergpflanzen.

$A$ Junges Pflänzchen vom fädigen Typus. $B-C$ Fertile knäuelige Zwergpflanzen. $D-E$ Übergangsformen. $F-G$ Fädige bzw. knäuelige $Z$ wergpflanze mit aufrechtem Faden. $H$ Teile aus älteren aufrechten Fäden mit Sporangien.

zahlreich auftreten, so machen sie im Vergleich zu der großen Anzahl der Zwergpflanzen im allgemeinen nur einen ganz geringen Bruchteil aus.

Um die Ursachen für die Entstehung der Giffordia-Pflanzen aufzuklären, wurden die Nachkommenschaften vieler einzelner Zwergpflanzen untersucht. Die reifen Pflänzchen schwärmten jeweils einzeln in einem Tropfen aus; nach dem Festsetzen der Schwärmer wurden die Pflänzchen entfernt und die Kulturschalen mit Nährlösung aufgefüllt. In mehreren Versuchsreihen wurden die Pflänzchen nochmals in Tropfen übertragen, um sie ein zweites oder ein drittes Mal ausschwärmen zu lassen und auf diese Weise mehrere Tochtergenerationen derselben $Z$ wergpflanze miteinander vergleichen zu können. Es stellte sich heraus, daß die Giffordia-Pflanzen in der Nachkommenschaft einer einzelnen Zwergpflanze auftraten. Das Verhältnis der langfädigen zu den Zwergpflanzen war ganz verschieden, es konnten die Zwergpflanzen oder die 


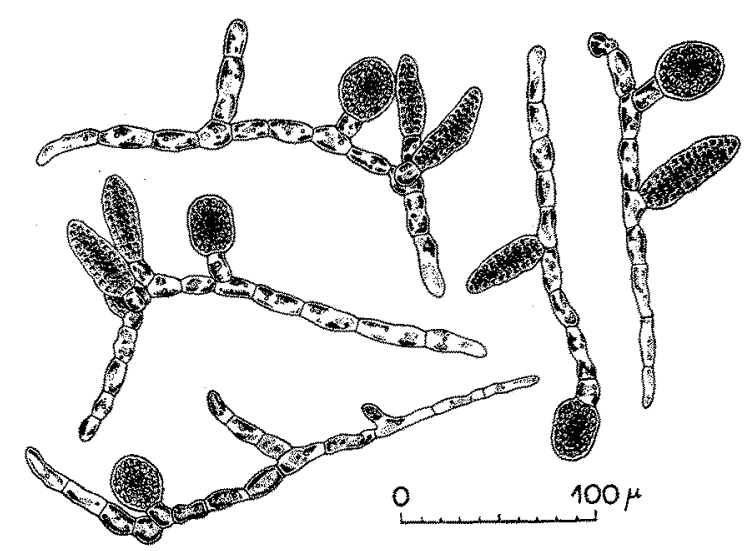

Abb. 5. Giffordia fuscata. Zwergpflanzen mit uni- und plurilokulären Sporangien (nach einer Photographie gezeichnet).

Giffordia-Pflanzen überwiegen, in einem Falle entwickelten sich sogar ausschließlich 26 Giffordia-Pflanzen. Die Tochtergenerationen ein- und desselben Zwergpflänzchens verhielten sich im allgemeinen gleichartig, doch kam es auch häufig vor, daß nur eine oder zwei von drei Nachkommenschaften langfädige Pflanzen enthielt.

Das Auftreten von Giffordia-Pflanzen in der Nachkommenschaft eines einzelnen Zwergpflänzchens machte es unwahrscheinlich, daß die langfädigen Pflanzen vielleicht doch einer übersehenen Gametenverschmelzung ihren Ursprung verdanken könnten. Schließlich hätte eine Kopulation der Schwärmer in dem Tropfen nicht der Beobachtung entgehen können. Trotzdem wurde eine weitere Versuchsreihe angestellt, die die Frage klären sollte: können Giffordia-Pflanzen auch in der Nachkommenschaft eines einzelnen Sporang iums entstehen? Für diese Versuche eigneten sich nur ganz junge Zwergpflänzchen vom fädigen Typus mit wenigen Sporangien, bei denen eine genaue Kontrolle der entleerten Sporangien möglich war. Tatsächlich trat in einem Falle eine Giffordia-Pflanze in der Nachkommenschaft eines einzelnen Sporangiums auf. Damit dürfte erwiesen sein, daß die Giffordia-Pflanzen einer Heteroblastie der Zwergpflanzen-Schwärmer ihre Entstehung verdanken.

Die Versuchsreihe führte zugleich zu dem weiteren Ergebnis, daß die Nachkommenschaften $f$ ädiger Zwergpflanzen überhaupt nur selten GiffordiaPflanzen enthielten. Es konnte durch parallele Versuchsreihen mit knäueligen und fädigen $Z$ wergpflanzen aus der gleichen Kultur bestätigt werden, daß die Giffordia-Generation vorzugsweise in der Nachkommenschaft knäueliger Zwergpflanzen auftrat.

Während die Kulturversuche bis zum Herbst 1953 keine weiteren Ergebnisse lieferten, wurde im Dezember eine überraschende Beobachtung gemacht: mehrere Kulturen enthielten Zwergpflanzen mit unilokulären Sporangien (Abb. 5). Der Anteil solcher Pflänzchen war nur gering, immerhin konnten aber aus einer Petrischale über 80 Zwergpflänzchen mit unilokulären Sporangien isoliert werden. Im allgemeinen trugen die jungen Pflänzchen gleichzeitig auch plurilokuläre Sporangien, manchmal saßen zwei unilokuläre Sporangien auf einer Zwergptlanze. In den Sporangien waren die Schwärmer mit ihrem Augenfleck deutlich zu erkennen, sie entleerten sich aber nur in ganz 
seltenen Fällen. Von 82 einzeln in Tropfen übertragenen Pflänzchen schwärmite nur ein einziges unilokuläres Sporangium aus, bei den übrigen erfolgte die Keimung im Inneren des Sporangiums. Auch in den ungestört wachsenden Kulturen wurde nur äußerst selten ein leeres unilokuläres Sporangium beobachtet. Aus den Schwärmern des einen entleerten Sporangiums entwickelten sich etwa 30 Zwergpflänzchen, die der Mutterpflanze ähnlich waren und plurilokuläre Sporangien trugen. In den anderen Fällen bildeten sich gallenartige dunkelbraune Klumpen auf den Zwergpflanzen, nachdem die Keimlinge die Wandung des unilokulären Sporangiums durchbrochen hatten. Häufig wuchs auch die Stielzelle eines unilokulären Sporangiums zu einem Faden aus, durchdrang das nicht entleerte Sporangium und endete mit einem plurilokulären Sporangium.

Schließlich wurde erst vor kurzem ein weiteres für die Deutung des Entwicklungszyklus von Giffordia fuscata wichtiges Ergebnis erzielt: ein geringer Bruchteil der Schwärmer eines unilokulären Sporangiums der GiffordiaPflanze kann sich unmittelbar zu der langfädigen Form entwickeln. Die in der Zwerggeneration festgestellte Heteroblastie tritt also bereits bei den Schwärmern des unilokulären Sporangiums auf, aus denen die Zwerggeneration entsteht. Für diesen Versuch wurden 88 Fadenstücke mit unilokulären Sporangien einzeln in Tropfen übertragen. In 25 Fällen entleerten sich die Sporangien, und die Schwärmer entwickelten sich. Drei von diesen Nachkommenschaften enthielten Giffordia-Pflanzen: in zwei Fällen war es je eine neben etwa $20 \mathrm{Zwergpflanzen,} \mathrm{im} \mathrm{dritten} \mathrm{Falle} \mathrm{entstanden} 6$ Giffordia-Büschel neben etwa $90 \mathrm{Zwergpllanzen.} \mathrm{Hier} \mathrm{hatten} \mathrm{allerdings} 2$ an einem Fadenstïck sitzende unilokuläre Sporangien ihre Schwärmer in den Tropfen entleert.

\section{Besprechung der Ergebnisse}

Das Vorkommen unilokulärer Sporangien an den Zwergpllanzen läßt den Lebenszyklus von Giffordia fuscata in einem völlig neuen Licht erscheinen. Ohne diese Beobachtung wäre es nicht so leicht gewesen, sich von der Vorstellung freizumachen, daß die Zwergpflanzen vielleicht doch als haploide Generation anzusehen wären. Es wäre dann zwar schwierig gewesen, die Entstehung der Diplophase zu erklären, denn eine geschlechtliche Funktion der Zwergpflanzenschwärmer war nicht nachzuweisen. Für die Entstehung der büscheligen Giffordia-Generation hätte man eine parthenogenetische Entwicklung einzelner Schwärmer im Sinne WinkLers (1942) annehmen müssen.

Das Auftreten unilokulärer Sporangien in beiden Generationen möchte ich in dem Sinne deuten, daß beide Generationen trotz ihrer morphologischen Verschiedenheit Diplonten sind und keine Reduktionsteilung in den unilokulären Sporangien erfolgt. Die beiden heteromorphen Generationen bestehen völlig unabhängig nebeneinander. Sie stehen nur durch die Heteroblastie ihrer Schwärmer in einem lockeren Zusammenhang. Sowohl die Schwärmer aus unilokulären Sporangien der Giffordia-Pllanze als auch diejenigen aus plurilokulären Sporangien der Zwerggeneration können beide Typen von Pflanzen erzeugen. Die nur selten auftretenden unilokulären Sporangien sind hier ebenso Organe der vegetativen Vermehrung wie die plurilokulären, durch deren Schwärmer sich beide Generationen im allgemeinen vermehren.

Die Erscheinung der Heteroblastie ist ebenso wie die morphologische 
Verschiedenheit der beiden homophasischen Generationen und die starke Variabilität der Zwergpflanzen nicht leicht zu verstehen. Die Versuche haben gezeigt, daß der Phänotypus der Zwergpflanzen durch Variation der äußeren Bedingungen - Zusammensetzung der Nährlösung - veränderlich ist. Auch das mehr oder weniger häufige Auftreten von Giffordia-Pflanzen in der Nachkommenschaft einzelner Zwergpflanzen deutet auf eine große Labilität der Faktoren hin, die die äußere Erscheinungsform bewirken. Heteroblastie wurde bei Phaeophyceen schon öfter beobachtet, z. B. bei Ascocyclus orbicularis (Sauvageau 1924); nach Kylin (1933) beruht sie hier auf äußeren Faktoren, indem die Schwärmer, die sich an der Wasseroberfläche ansetzen, immer zu ectocarpoiden Fäden auswachsen, während sie sich auf dem Boden der Kulturschale zu Scheiben entwickeln. Das gleiche Verhalten fand Kruin (1933) auch bei den Schwärmern von Eudesme zosterae, während SAuvageaus (1927) Beobachtungen schließen lassen, daß die Heteroblastie nicht nur durch äußere Ursachen bedingt ist. Die von Hollenserg (1941) bei Haplerophycus canaliculatus beobachtete Erscheinung kann nicht als Heteroblastie im eigentlichen Sinne angesehen werden, wenn sie auf Schwärmer mit dem einfachen bzw. doppelten Chromosomensatz zurückgeht.

Bei einer ganzen Reihe von Braunalgen führten entwidklungsgeschichtliche Untersuchungen zu dem Schluß, daß keine Reduktionsteilung im unilokulären Sporangium stattfindet. Für Pylaiella rupincola hat KYLIN (1937) diese Annahme auf Grund der Kulturversuche von Dammann (1930) und eigener Beobachtungen an Freilandmaterial wahrscheinlich gemacht. Bei Asperococcus compressus, Dictyosiphon chordaria, Striaria attenuata oder Elachista fucicola führten Kulturversuche zu der gleichen Folgerung. In diesen Fällen tritt keine selbständig sich vermehrende mikroskopische Generation auf, es erscheinen auch niemals plurilokuläre Sporangien.

Einer eingehenderen Betrachtung müssen wir die entwicklungsgeschichtlichen Untersuchungen an Eudesme zosterae unterziehen. SAuvagEaus (1927) Ergebnisse wurden von KYLIN (1933) bestätigt. Diese Alge besitzt pluri- und unilokuläre Sporangien. Beiderlei Schwärmer entwickeln sich in gleicher Weise zu einer aus Myrionema- und Streblonema-Stadien gemischten Generation. KyLIN schließt aus diesem gleichartigen Verhalten, daß beide Schwärmersorten diploid sein könnten und daß die Reduktionsteilung im unilokulären Sporangium unterblieben sein könnte. $\mathrm{Zu}$ neuen Eudesme-Pflanzen gelangte KyLIN nur in einem Versuch, bei dem er von Thallusstücken ausging, die ausschließlich plurilokuläre Sporangien trugen. Innerhalb von etwa 4 Wochen entwickelte sich eine fertile Generation, nach weiteren 5 Wochen waren bis zu $2 \mathrm{~mm}$ hohe Eudesme-Pflanzen vorhanden. Das Eudesme-Stadium ist eine vegetative Weiterentwicklung der Assimilationsfäden des Myrionema-Stadiums. Ich sehe darin eine starke Stütze für die vermutete Diploidie dieser Generation.

Die Untersuchungen von Birgithe Ruud Föyn (1934) an Eudesme zosterae sind im Zusammenhang mit meinen Versuchen bedeutsamer. Hier dienten Schwärmer aus unilokulären Sporangien als Ausgangsmaterial und führten wie in Kyurns Versuchen zu Myrionema- und Streblonema-Stadien mit plurilokulären Sporangien. Die Schwärmer entwickelten sich ohne Kopulation und ergaben wiederum ähnliche Folgegenerationen. In zwei Kulturen traten aber auch Eudesme-Pflanzen mit pluri- und unilokulären Sporangien auf. Diese Entwicklung stimmt also mit der bei Giffordia fuscata überein.

4 Mecresuntersuchungen Bd. V. H. 1 
Schließlich verdienen auch die Kulturversuche an Elachista stellaris in den Kreis dieser Betrachtung gezogen zu werden, aus denen KyLin (1937) zu dem Schluß gelangte, daß keine Reduktionsteilung im unilokulären Sporangium stattfindet. Aus den Schwärmern entwickelten sich kriechende Fäden, die plurilokuläre Sporangien und neue Elachista-Pflanzen trugen. Die Schwärmer dieser plurilokulären Sporangien wiederholten die Entwicklung. Es ist nur ein kieiner Schritt, um die Entstehung von Giffordia-Pflanzen in der Zwerggeneration von diesem Entwicklungsschema abzuleiten: die Giffordia-Pflanzen entstehen nicht direkt aus einem sporangientragenden Kriechfaden, sondern aus einem sterilen Rhizoid, zu dem sich ein Teil der Zwergpflanzen-Schwärmer entwickelt. Der gleiche Unterschied besteht übrigens auch gegenüber Eudesme zosterae, wo sich die Pflanze aus den Assimilationsfäden des fertilen $M y$ rionema-Stadiums entwickelt.

Die an Giffordia fuscata erzielten Ergebnisse können nicht ohne Rückwirkung auf ähnlich gelagerte Beispiele in der Entwicklung mancher anderer Ectocarpalen bleiben. In den meisten Fällen entwickelt sich aus den Schwärmern der unilokulären Sporangien eine mikroskopisch kleine Generation. Nur selten gelang es, ihre Gametophytennatur durch die Beobachtung der Fusion ihrer Schwärmer nachzuweisen (Mesogloia vermiculata, PARKe 1933, Phloeospora brachiata, Mathias 1935) und die Form der Ausgangspflanze in den Kulturen wieder zu erhalten. In vielen Fällen aber mußte der Analogieschluß diese Beobachtungslücken ausfüllen. Ich greife als Beispiele nur heraus: Litosiphon pusillus, Myrionema strangulans, Stilophora rhizodes. Nach den hier mitgeteilten Ergebnissen scheint es mir nicht ratsam, bei diesen und manchen anderen Formen einen heterophasischen Generationswechsel anzunehmen, solange keine lückenlosen Kulturversuche oder zytologischen Beweise vorliegen.

Heteromorphe Generationen sind im Lebenszyklus einer Ectocarpacee nicht mehr ganz unerwartet, nachdem bei Acinetospora eine antheridientragende $Z$ werggeneration gefunden wurde (KoRNmanN 1953). Es überrascht aber, daß diese Erscheinung bei einer Gattung auftritt, die in Giffordia secunda eine Art mit anisogamer Befruchtung und - sehr wahrscheinlich - isomorphem Generationswechsel aufweist. Das abweichende Verhalten von Giffordia fuscata wird wohl mit der fehlenden Reduktionsteilung zusammenhängen.

Feldmann (1952) hat kürzlich auf die Möglichkeiten einer stammesgeschichtlichen Differenzierung hingewiesen, indem heteromorphe Partner eines Entwicklungszyklus sich getrennt und zu selbständigen Formen weiterentwickelt haben könnten. Als Beispiele führt er die Entwicklungszyklen von Codiolum-Urospora und Derbesia-Halicystis an. Für einzelne Arten dieser Gattungen ist der entwicklungsgeschichtliche Zusammenhang erwiesen. Es gibt aber in beiden Formenkreisen auch Arten, die des entsprechenden Partners entbehren. Der Fortfall der Gametenverschmelzung oder der Reduktionsteilung kann wahlweise zu der Entstehung selbständiger Formen geführt haben.

Die bei Giffordia fuscata angetroffenen Verhältnisse bereichern die Hypothese Frodmanns um ein weiteres lehrreiches Beispiel. Hier stehen die beiden Partner eines Entwicklungszyklus nur noch in einem ganz lockeren Zusammenhang. Unilokuläre Sporangien in beiden Generationen betonen ihre Selbständigkeit. Das Auftreten unilokulärer Sporangien an den Zwergpflanzen ist eigentlich gar nichts Besonderes, da schon die übrigen Beobachtungen - fehlende Sexualität, Heteroblastie ihrer Schwärmer - zu der Annahme berech- 
tigen, diese Generation als diploid anzusehen. In diesem Zusammenhang kommt der Vermutung Parkes (1933) eine besondere Bedeutung zu, daß Ascocyclus ein Stadium im Formenkreis von Eudesme zosterae darstellen könnte. Frirsch (1945 p. 138) hält eine Zusammengehörigkeit für unwahrscheinlich, nachdem unilokuläre Sporangien bei Ascocyclus condhicola gefunden worden sind (Feldmann 1937); er hält daher Ascocyclus für einen selbständigen Diplonten. Ich habe weiter oben ausgeführt, daß die Entwicklung von Eudesme zosterae mit der von Giffordia fuscata viele Ahnlichkeiten aufweist. Das gleichartige Auftreten unilokulärer Sporangien bei Ascocyclus und den Zwergpflanzen von Giffordia fuscata möchte ich als weitere Übereinstimmung deuten, die zugunsten der Annahme von PARke spricht. Und sollte schließlich die übereinstimmende Heteroblastie bei Eudesme und Ascocyclus ein reiner Zufall sein?

Nach den obigen Ausführungen halte ich es für durchaus wahrscheinlich, daß viele der im Freien vorkommenden mikroskopischen Braunalgen selbständig gewordene Partner ehemaliger oder noch bestehender Lebenszyklen sein könnten. Die Zwerggeneration von Giffordia fuscata wäre - falls man sie im Freien gefunden hätte - zweifellos als selbständige Art betrachtet worden. Möglicherweise könnten auch die uns aus Kulturen bekannten mikroskopischen Generationen vieler Arten unter den Bedingungen des natürlichen Standorts in etwas abgeänderter Tracht erscheinen.

Auf eine andere Möglichkeit der Entstehung selbständiger Partner aus einem ursprünglichen Entwicklungszyklus möchte ich zum Schluß noch hinweisen. Sie könnte über Formen führen, die Gametangien neben Organen ungeschlechtlicher Vermehrung ausbilden. Ich denke dabei an die in meinen Kulturen von Acinetospora crinita aufgetretene Zwerggeneration mit Antheridien (Kornmann 1953). Eine ganz ähnliche im Freien gefundene Form hat Howe (1914) als Streblonema Cokeri beschrieben. Meine Kulturen enthielten häufig Pflänzchen, die nur ungeschlechtliche plurilokuläre Sporangien trugen. Man kann sich vorstellen, daß der Wegfall der Antheridien zu einer selbständigen streblonemoiden Form führen könnte.

Meinem technischen Assistenten, Herrn Paul Heinz Sarring, danke ich für die Mitwirkung bei der Durchführung der zahlreichen Kulturversuche sowie die Anfertigung der Zeichnungen.

\section{Zusammenfassung}

1. Der Entwicklungszyklus von Giffordia fuscata umfaßt zwei heteromorphe Generationen, die durch die Heteroblastie ihrer Schwärmer in einem lockeren Zusammenhang stehen:

a) Die langfädige Giffordia fuscata, die sich durch die Schwärmer ihrer plurilokulären Sporangien selbständig vegetativ vermehren kann. Die Schwärmer ihrer unilokulären Sporangien zeigen Heteroblastie; die Mehrzahl ergibt Zwergpflanzen, ein kleiner Teil entwickelt sich unmittelbar zu der büscheligen Form.

b) Die Zwerggeneration, die reichlich plurilokuläre und ganz selten unilokuläre Sporangien trägt. Die Schwärmer der plurilokulären Sporangien sind heteroblastisch, die meisten ergeben wieder Zwergpflanzen, so daß sich die Zwerggeneration selbständig vegetativ erhalten kann. Ein kleiner Bruchteil der Schwärmer entwickelt sich zu der büscheligen Giffordia-Pflanze. 
2. Irgendwelche Anzeichen für eine Sexualität der Zwerggeneration sind nicht vorhanden; Giffordia-Pflanzen können aus den Schwärmern eines einzelnen Zwergpflänzchens und sogar eines einzelnen Sporangiums entstehen.

3. Das Vorkommen unilokulärer Sporangien an den Zwergpflanzen, die fehlende Sexualität der Zwerggeneration und die Heteroblastie in beiden Generationen berechtigen zu der Annahme, die beiden heteromorphen Generationen als Diplonten anzusehen.

4. Es wird auf ähnliche Beziehungen in der Entwicklung von Eudesme zosterae zu Ascocyclus hingewiesen sowie auf die Möglichkeit, daß viele mikroskopische Braunalgen selbständig gewordene Partner eines früheren oder noch bestehenden Lebenszyklus sein könnten.

\section{Schriftum}

Dammann, Hildegard, 1930. Entwicklungsgeschichtliche und zytologische Untersuchungen an Helgoländer Meeresalgen. Wiss. Meeresunters. N. F. Abt. Helgol. 18

Feldmann, J., 1937. Les Algues marines de la côte des Albères. I-III. Cyanophycées, Chlorophycées, Phéophycées. Rev. Algol. 9.

- 1952. Les cycles de reproduction des Algues et leurs rapports avec la phylogénie. Rev. de Cytol. et de Biol. végét. 13.

Föyn, B. Ruud, 1934. Über den Lebenszyklus einiger Braunalgen. Bergens Mus. Årbok, Naturvitensk. rekke Nr. 2

Fritsch, F. E., 1945. The structure and reproduction of the algae. Vol. II. Cambridge.

Hollenberg, G. J., 1941. Culture studies of marine algae. II. Hapterophycus canaliculatus S. \& G. Amer. Jour. Bot. 28.

Howe, M. A., 1914. The marine algae of Peru. Mem. Torrey Bot. Club. 15.

Kornmann, P., 1953. Dic Entwicklung von Giffordia fuscata (Zan.). Proc. First Intern. Seaweed Symposium, Edinburgh 1952. Inst. of Seawced Res., Inveresk, Midlothian, Scotl.

- 1953. Der Formenkreis von Acinetospora crinita (Carm.) nov. comb. Helgol. Wiss. Meeresunters. 4.

Kuckuck + , P., 1953. Ectocarpaceen-Studien I. Hecatonema, Chilionema, Compsonema. Helgol. Wiss. Meeresunters. 4 (herausgeg. von P. Kornmann).

Ky l in, H., 1933. Über die Entwicklungsgeschichte der Phaeophyceen. Lunds Univ. Årsskrift, N. F. Avd. 2, 29.

- 1937. Bemerkungen über die Entwicklungsgeschichte einiger Phaeophyceen. Ebenda, 33.

- 1947. Dic Phaeophyceen der schwedischen Westküste. Ebenda, 43.

Mathias, W. T., 1935. The life-history and cytology of Phloeospora brachiata Born. Public. Hartley Bot. Lab. Univ. Liverpool, N.r. 13.

Parke, Mary, 1933. A contribution to knowledge of the Mesogloiaceae and associated families. Ebenda, Nr. 9

Sa uva geau, C., 1897. Sur quelques Myrionémacées. Ann. Sci. nat., 8. série, Bot. 5.

- 1927. Sur le Castagnea Zosterae Thur. Bull. Stat. biol. d'Arcachon. 24.

Winkler, H., 1942. Uber den Biontenwechsel und die Abweichungen von seinem normalen Verlauf. Planta. 33. 